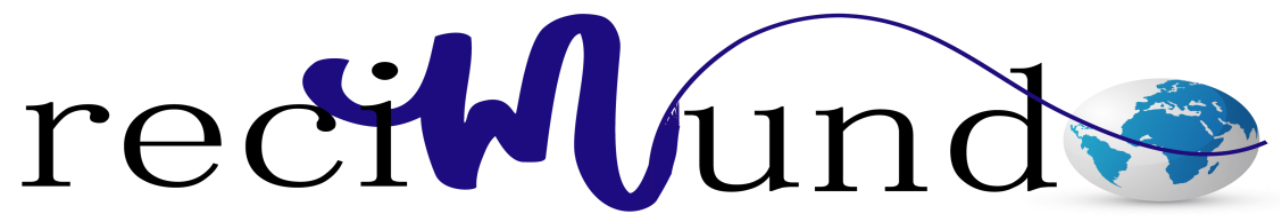

Revista Científica Mundo de la Investigación y el Conocimiento

Hugo Jaime Vargas Marín a; Evelin Edda Pinargote Junco b; Alexis Eduardo Verdesoto Arguello ${ }^{\mathrm{c}}$

Elaboración de criterios al diseñar objetos virtuales de aprendizaje como estrategia metodológica en entornos virtuales y su aporte al trabajo cooperativo en el aula Revista Científica Mundo de la Investigación y el Conocimiento. Vol. 2 núm., 1, febrero, ISSN: 2588-073X, 2018, pp. 117-129

DOI: $10.26820 /$ recimundo/2.1.2018.117-129

Editorial Saberes del Conocimiento

Recibido: 05/12/2017

Aceptado: 10/02/2018

a. Ingeniero en Sistemas; Licenciado en Ciencias de la Educación Especialización Educación de Adultos; Técnico Superior en Programación de Sistemas de Computo; Tecnólogo en Informática - Análisis de Sistemas; Docente del Instituto Tecnológico Superior "Aguirre Abad"; jvargas@itsaa.edu.ec

b. Licenciada en ciencias de la educación mención Informática; Profesora de segunda enseñanza esp. Informática; Tecnóloga pedagógica en Informática; Docente del Instituto Tecnológico Superior Juan Bautista Aguirre; evelinpjunco81@gmail.com

c. Ingeniero en Sistemas e Informática; Docente del Instituto Técnico Superior Eugenio Espejo; alexisverdesoto@gmail.com 


\section{Elaboración de criterios al diseñar objetos virtuales de aprendizaje como estrategia metodológica en entornos virtuales y su aporte al trabajo cooperativo en el aula}

Vol. 2, núm. 1., (2018)

Hugo Jaime Vargas Marín; Evelin Edda Pinargote Junco; Alexis Eduardo Verdesoto Arguello

\section{RESUMEN}

Objetivo: La presente revisión tiene como objetivo conocer los criterios básicos para elaborar OVAs, mediante un estudio de caso que permita determinar su incidencia como estrategia metodológica aplicada en un entorno virtual por los docentes del Instituto Tecnológico Superior Aguirre Abad. Metodología: Se realizó una búsqueda con una retrospectiva de 10 años de antigüedad; en revistas de alto impacto y en bases científicas digitales como: Scielo; Cochrane; EBSCO; Redalyc. Incluyendo también libros académicos. La mayoría correspondió al idioma Ingles; aunque también se incluyó en menor porcentaje en español. Conclusiones: Conocer y diseñar criterios de evaluación de un OVA en la enseñanza virtual, aplicando los modelos de diseño instruccional ADDIE y ASSURE. Evaluar el OVA con una rúbrica como estrategia para medir el nivel de captación del estudiante en la enseñanza virtual.

Palabras clave: Internet, Objetos Virtuales de aprendizaje (OVA), Tecnología de la Información y Comunicación (TIC), Entornos Virtuales de Aprendizaje (EVA), Entornos Virtuales de Enseñanza-Aprendizaje (EVEA). 


\title{
Elaboración de criterios al diseñar objetos virtuales de aprendizaje como estrategia metodológica en entornos virtuales y su aporte al trabajo cooperativo en el aula
}

Vol. 2, núm. 1., (2018)

Hugo Jaime Vargas Marín; Evelin Edda Pinargote Junco; Alexis Eduardo Verdesoto Arguello

\begin{abstract}
Objective: The objective of this review is to know the basic criteria for preparing OVAs, by means of a case study that allows determining its incidence as a methodological strategy applied in a virtual environment by the teachers of the Aguirre Abad Higher Technological Institute. Methodology: A search was conducted with a retrospective of 10 years old; in high impact journals and on digital scientific bases such as: Scielo; Cochrane; EBSCO; Redalyc. Including also academic books. The majority corresponded to the English language; although it was also included in a lower percentage in Spanish. Conclusions: Know and design evaluation criteria of an OVA in virtual teaching, applying the instructional design models ADDIE and ASSURE. Evaluate the OVA with a rubric as a strategy to measure the level of student acquisition in virtual teaching.
\end{abstract}

Keywords: Internet, Virtual Learning Objects (OVA), Information and Communication Technology (ICT), Virtual Learning Environments (EVA), Virtual Teaching-Learning Environments (EVEA). 


\section{Elaboración de criterios al diseñar objetos virtuales de aprendizaje como estrategia metodológica en entornos virtuales $y$ su aporte al trabajo cooperativo en el aula}

Vol. 2, núm. 1., (2018)

Hugo Jaime Vargas Marín; Evelin Edda Pinargote Junco; Alexis Eduardo Verdesoto Arguello

\section{Introducción.}

El internet representa la mayor biblioteca virtual del mundo. Según datos de la ONU los países con mayor acceso a Internet están todos en Asia y el Medio Oriente. La República de Corea sigue primero esa lista, con un $98.5 \%$ de sus hogares conectados. Islandia por su parte, tiene el mayor porcentaje de personas que utilizan Internet, con $98.2 \%$. (BROADBAND COMMISSION FOR SUSTAIMABLE DEVELOPMENT, 2016) En Latinoamérica estudios estadísticos de conexión individual al internet de la Unión Internacional de Telecomunicaciones, sitúan a Chile en primer lugar con el $53.9 \%$ de internautas, mientras que Nicaragua con 10.6\% es el país menos conectado a la red. Ecuador se encuentra en el puesto 95 con el $31.4 \%$ de la población accediendo al internet. (INEC, 2013)

Estas aseveraciones estadísticas determinan un alto consumo de internet en Latinoamérica, con especial connotación en Ecuador. No obstante, el tiempo de navegación debe ser eficiente para mejorar el proceso educativo. En la actualidad existe todo tipo de datos; genéricos, fundamentales, médicos, noticias, Por Ej: Biblioteca Nacional de Medicina de los Estados Unidos; The New York Times, etc. Comúnmente estos datos son de fácil acceso para las personas dado a que las actuales páginas web están diseñadas con los formatos adecuados para que éstas puedan acceder a la información, en este proceso se suscitan inconvenientes relacionados con la excesiva cantidad de datos, lo que imposibilita su manipulación. 


\section{Elaboración de criterios al diseñar objetos virtuales de aprendizaje como estrategia metodológica en entornos virtuales y su aporte al trabajo cooperativo en el aula}

Vol. 2, núm. 1., (2018)

Hugo Jaime Vargas Marín; Evelin Edda Pinargote Junco; Alexis Eduardo Verdesoto Arguello

Nos encontramos en un momento en que la sociedad avanza y el uso de la tecnología se ve reflejado en todas partes, por ende es de mucha importancia en todos los ámbitos de la vida y principalmente en el educativo.

Este nuevo panorama da origen por ejemplo, a la aparición de los llamados Entornos Virtuales de Aprendizaje o EVA, los cuales forman parte de las tendencias educacionales actuales.

En este marco se cuestiona ¿Cómo pueden aportar los entornos virtuales de aprendizaje en el trabajo cooperativo en el aula?, siendo este el reto al que nos enfrentamos los docentes al tratar de incorporar nuevas tecnologías a nuestra práctica docente.

La presente revisión tiene como objetivo conocer los criterios básicos para elaborar OVAs, mediante un estudio de caso que permita determinar su incidencia como estrategia metodológica aplicada en un entorno virtual por los docentes del Instituto Tecnológico Superior Aguirre Abad.

\section{Metodología.}

Se realizó una búsqueda con una retrospectiva de 10 años de antigüedad; en revistas de alto impacto y en bases científicas digitales como: Scielo; Cochrane; EBSCO; Redalyc. Incluyendo también libros académicos. La mayoría correspondió al idioma Ingles; aunque también se incluyó en menor porcentaje en español. 


\section{Elaboración de criterios al diseñar objetos virtuales de aprendizaje como estrategia metodológica en entornos virtuales $y$ su aporte al trabajo cooperativo en el aula}

Vol. 2, núm. 1., (2018)

Hugo Jaime Vargas Marín; Evelin Edda Pinargote Junco; Alexis Eduardo Verdesoto Arguello

\section{Revisión de la literatura}

Las plataformas virtuales aplicadas en pedagogía han experimentado una evolución exponencial en las últimas décadas. Trece de cada veinte universidades en América Latina adaptan sus procesos pedagógicos (planeamiento curricular, la impartición de contenidos, la evaluación, etc.) al uso de plataformas dinámicas, multiusuarios y autonómicas fortaleciendo el proceso áulico. (CEPAL, 2016) Estas herramientas adquieren más relevancia debido a que contribuyen a modificar los objetivos y a revisar los planteamientos docentes actuales los estudiantes ya no solo tienen que ser buenos conocedores de cada materia en cuestión, sino también deben desarrollar otras actitudes como la creatividad, el espíritu crítico y la capacidad para el aprendizaje continuo que la sociedad les depara. (Mora Mora, y otros, 2015)

(Zimmer, 2008) determinó que las herramientas docentes 2.0 permiten la construcción de estrategias colaborativas para mejorar el proceso de docencia aprendizaje para los estudiantes en los aspectos mencionados. (Grosseck, 2009) según el autor este desarrollo debe guiar nuevas técnicas pedagógicas basadas en la colaboración y en la creación de inteligencia colectiva entre los estudiantes. En este aspecto, también hay voces críticas que resaltan los riesgos del uso de este tipo de plataformas entre los más jóvenes. (Mora Mora, y otros, 2015)

Cuando los estudiantes colaboran para construir conocimiento no está claro qué aporta cada uno ni tampoco lo que aprende en esas circunstancias. Por este motivo la supervisión del alumno por los profesores se convierte en un aspecto crítico cuando estas herramientas se utilizan en el proceso docente. 


\section{Elaboración de criterios al diseñar objetos virtuales de aprendizaje como estrategia metodológica en entornos virtuales y su aporte al trabajo cooperativo en el aula}

Vol. 2, núm. 1., (2018)

Hugo Jaime Vargas Marín; Evelin Edda Pinargote Junco; Alexis Eduardo Verdesoto Arguello

La importancia sustancial del uso de las herramientas e-learning se ejemplifica en esta recopilación bibliográfica. Su estudio, análisis y perspectivas en torno a su aplicación en el currículo educativo ecuatoriano son imprescindibles e irrefutables.

Sin duda las TIC como motor de cambio e innovación de los procesos y escenarios educativos, juegan un rol fundamental ya que casi todas las actividades educativas giran en torno al uso frecuente de las tecnologías. Lo que han permitido que en el ámbito educativo se innove de tal manera que el proceso enseñanza-aprendizaje se desarrolle de manera más dinámica, por tanto se plantean nuevas estrategias metodológicas a disposición de los docentes como son los OVA (Objetos Virtuales de Aprendizaje).

A través del tiempo esta temática ha sido el objeto de estudio en muchas investigaciones, que analizadas por los expertos desde distintas ópticas han generado interrogantes que pretenden comprender y definir su importancia dentro de la educación. Tenemos por ejemplo a (Castañeda López, 2014), el cual expone que "los OVA son solamente una herramienta educativa que puede insertarse en propuestas curriculares y metodologías de enseñanza y aprendizaje”.

De la misma manera está (Silva Quiceno \& Sosa, 2016) en la Universidad Cooperativa de Colombia, manifiesta que "en la actualidad los OVA son de suma importancia en los modelos pedagógicos y en los programas por competencias, ya que permiten una interacción entre profesor y estudiante para el fortalecimiento del proceso enseñanza aprendizaje y la aplicación de los conocimientos de la clase magistral por parte del estudiante para el logro de las 


\section{Elaboración de criterios al diseñar objetos virtuales de aprendizaje como estrategia metodológica en entornos virtuales $y$ su aporte al trabajo cooperativo en el aula}

Vol. 2, núm. 1., (2018)

Hugo Jaime Vargas Marín; Evelin Edda Pinargote Junco; Alexis Eduardo Verdesoto Arguello

competencias del curso.", dando a entender que esta herramienta permite contribuir al mejoramiento de la calidad educativa.

En estos últimos años la educación ha utilizado los entornos virtuales y de esta manera ha mejorado el aprendizaje a través de plataformas virtuales las cuales incluyen varias herramientas para fortalecer el aprendizaje, según como lo menciona (Obando Bastidas, 2015) "Lo importante de un objeto virtual de aprendizaje es que cumpla con su propósito de formación y que fortalezca de alguna manera el aprendizaje".

La progresiva desformalización de los sistemas educativos por estar en auge la educación virtual o a distancia, ha ocasionado que las instituciones educativas implementen entornos virtuales de aprendizajes y estas envíen a sus docentes a capacitarse para ser más competitivos en las aulas. Al hablar de EVA (Entornos Virtuales de Aprendizaje), menciona (Belloch, 2012) “el e-learning no trata solamente de tomar un curso y colocarlo en un ordenador, se trata de una combinación de recursos, interactividad, apoyo y actividades de aprendizaje estructuradas. Para realizar todo este proceso es necesario conocer las posibilidades y limitaciones que el soporte informático o plataforma virtual nos ofrece. ”, Concluye.

Un EVEA posee las siguientes características (Salinas, 2011):

- Ambiente electrónico, creado y constituido por tecnologías digitales.

- Hospedado en la red y se puede tener acceso remoto a sus contenidos a través de algún tipo de dispositivo con conexión a Internet. 


\section{Elaboración de criterios al diseñar objetos virtuales de aprendizaje como estrategia metodológica en entornos virtuales y su aporte al trabajo cooperativo en el aula}

Vol. 2, núm. 1., (2018)

Hugo Jaime Vargas Marín; Evelin Edda Pinargote Junco; Alexis Eduardo Verdesoto Arguello

- Las aplicaciones o programas informáticos que lo conforman sirven de soporte para las actividades formativas de docentes y estudiantes.

- La relación didáctica no se produce en ellos "cara a cara" (como en la enseñanza presencial), sino mediada por tecnologías digitales

- Acceso independiente de tiempo y espacio geográfico.

Los entornos personales de aprendizaje se presentan como un sistema bisagra donde integrar el entorno virtual institucional en el que estamos distribuyendo cursos y asociado preferentemente al aprendizaje formal, y este entorno más informal que ofrecen redes sociales y comunidades virtuales de aprendizaje para construir las propias Redes Personales de Conocimiento (PKN). (Salinas J., 2013)

Como elementos generales que configurarán la sociedad en las dos próximas décadas por la globalización, están el aprendizaje autónomo y personalizado a través de los LMS y el uso constante de las herramientas Web 2,0 así como los OVAs como complemento multimedia, es por esto que "la práctica educativa moldea el uso y la puesta en acción de la tecnología, la evoluciona y la convierte en parte indisociable de la práctica. Dicho de otro modo, las creencias y actitudes de los docentes sobre la enseñanza y el aprendizaje y la tecnología determinan lo que los docentes hacen con las TIC, pero dichas creencias y actitudes se elaboran y desarrollan especialmente mediante el uso de las TIC". (Adell \& Castañeda, 2012) 


\section{Elaboración de criterios al diseñar objetos virtuales de aprendizaje como estrategia metodológica en entornos virtuales $y$ su aporte al trabajo cooperativo en el aula}

Vol. 2, núm. 1., (2018)

Hugo Jaime Vargas Marín; Evelin Edda Pinargote Junco; Alexis Eduardo Verdesoto Arguello

Por otro lado, las tendencias educativas han cambiado con el transcurrir del tiempo, siendo así que de pasar de una educación de manera tradicional en donde el docente era el único protagonista en el aula, ahora vivimos en la era tecnológica en donde la educación constructivista va de la mano con el conectivismo que apunta hacia el buen uso de la misma y esto se ve reflejado en los llamados entornos virtuales de aprendizaje, los cuales son tendencia en la educación actual y están aportando al mejoramiento del trabajo cooperativo en el aula.

En efecto, "un entorno virtual de aprendizaje es un espacio educativo alojado en la web, conformado por un conjunto de herramientas informáticas que posibilitan la interacción didáctica” (Belloch, 2012), pero ¿Por qué se consideran a los EVA de utilidad para el proceso enseñanza-aprendizaje?, pues eso nos refieren (Bautista, Borges, \& Forés, 2006) cuando expresan "Los entornos virtuales de aprendizaje proporcionan más flexibilidad al estudiante, permitiéndole estudiar en cualquier momento", atribuyendo su importancia a la ayuda educativa que genera al permitir intercambiar conocimientos por medio de la tecnología, en base a las múltiples ventajas que se generan al utilizar esta modalidad de estudio.

Dicho esto, una característica principal que tienen los entornos virtuales de aprendizaje es que son elaborados pensando también en que deben generar trabajo cooperativo. Esto lo sustentan (Bustos Sánchez \& Coll Salvador, 2010) cuando expresan que las tecnologías "pueden llegar a comportar una modificación sustancial de los entornos de enseñanza y aprendizaje" y eso lo vemos reflejado en los entornos virtuales de aprendizaje porque permiten modificar aspectos psicológicos de las personas, tal como su manera de pensar y actuar en trabajos que impliquen relacionarse con otros, incluso a trabajar cooperativa o colaborativamente. 


\section{Elaboración de criterios al diseñar objetos virtuales de aprendizaje como estrategia metodológica en entornos virtuales y su aporte al trabajo cooperativo en el aula}

Vol. 2, núm. 1., (2018)

Hugo Jaime Vargas Marín; Evelin Edda Pinargote Junco; Alexis Eduardo Verdesoto Arguello

Entonces ¿Cómo aportan los entornos virtuales de aprendizaje al trabajo cooperativo en el aula? Al hablar de trabajo cooperativo citamos a (Guitert \& Giménez, 2000) quienes expresan que se da cuando "existe una reciprocidad entre un conjunto de individuos que saben diferenciar y contrastar sus puntos de vista de tal manera que llegan a generar un proceso de construcción de conocimiento", manifestándose principalmente que el trabajo cooperativo se fundamenta en un objetivo en común para todos los participantes de la actividad propuesta y manteniendo elementos esenciales como son: actitud ética, estrategias de planificación, resolución compartida de problemas, etc., que en general les permitirán trabajar en equipo a los participantes del entorno virtual, generando así el trabajo cooperativo final.

El uso de entornos virtuales de aprendizaje ha tenido muy buena aceptación por parte de los usuarios, sobre todo por el mundo tecnológico en que nos desenvolvemos hoy en día, facilitando así la adaptación a trabajos de manera conjunta, pues en su mayoría se encuentran diseñados para que generen esta competencia en los estudiantes, es decir la competencia del trabajo cooperativo en el aula.

\section{Conclusiones.}

Los entornos virtuales de aprendizaje, sin lugar a duda son un buen aliado al momento de querer innovar el quehacer educativo y en los actuales momentos contribuye al trabajo cooperativo en el aula. Se considera que tanto docentes, estudiantes y comunidad educativa en general evidencian desde cualquier otro lugar el desarrollo de su trabajo formativo, dando lugar a la nueva era tecnológica. 


\section{Elaboración de criterios al diseñar objetos virtuales de aprendizaje como estrategia metodológica en entornos virtuales $y$ su aporte al trabajo cooperativo en el aula}

Vol. 2, núm. 1., (2018)

Hugo Jaime Vargas Marín; Evelin Edda Pinargote Junco; Alexis Eduardo Verdesoto Arguello

La flexibilidad es una de las principales características que poseen los EVA, y esto favorece al planear actividades que potencien el trabajo cooperativo.

La implementación de las e-learning brinda mayores posibilidades de éxito en el proceso de aprendizaje, permitiendo acceso a clases originales, divertidas y significativas, además de una aplicación significativa.

El diseño educativo del currículo moderno requiere indispensablemente de adaptaciones curriculares contemporáneas como el uso de plataformas virtuales que faciliten la apropiación de los contenidos en un ambiente de aprendizaje cooperativo.

Por la versatilidad en cuanto a la planeación de actividades que tenemos a disposición los docentes en entornos virtuales de aprendizaje, la comunicación entre estudiantes al realizar trabajos grupales se ve mejorada, según la modalidad de educación actual.

Está comprobado en varios estudios que las nuevos enfoques educativos buscan la comunicación integral de diferentes métodos y tecnologías, fortaleciendo la comunicación efectiva, tal como lo hacen los entornos virtuales de aprendizaje.

\section{Bibliografía.}

Adell, J., \& Castañeda, L. (2012). Tecnologías emergentes, ¿pedagogías emergentes? Barcelona: Asociación Espiral, Educación y Tecnología.

Bautista, G., Borges, F., \& Forés, A. (2006). Didáctica universitaria en Entornos virtuales de enseñanza-aprendizaje. Madrid: Ediciones Narcea.

Belloch, C. (2012). Entornos Virtuales de Aprendizaje. Valencia: Universidad de Valencia. 


\section{Elaboración de criterios al diseñar objetos virtuales de aprendizaje como estrategia metodológica en entornos virtuales y su aporte al trabajo cooperativo en el aula}

Vol. 2, núm. 1., (2018)

Hugo Jaime Vargas Marín; Evelin Edda Pinargote Junco; Alexis Eduardo Verdesoto Arguello

BROADBAND COMMISSION FOR SUSTAIMABLE DEVELOPMENT. (2016). The Stare of Broadband: Broadband catalyzing sustainable development. Ginebra: ONU.

Bustos Sánchez, A., \& Coll Salvador, C. (2010). Los entornos virtuales como espacios de enseñanza y aprendizaje: una perspectiva psicoeducativa para su caracterización y análisis. Revista Mexicana de Investigación Educativa, 44(15), 163-194.

Castañeda López, D. (2014). Objeto virtual de aprendizaje como estrategia para la enseñanza de la materia y sus propiedades en los estudiantes de grado $10^{\circ}$. Manizales: Universidad Nacional de Colombia.

CEPAL. (2016). Estudio Económico de América Latina y el Caribe 2016: La Agenda 2030 para el Desarrollo Sostenible y los desafíos del financiamiento para el desarrollo. Santiago de Chile: Comisión Económica para América Latina y el Caribe.

Grosseck, G. (2009). To use or not to use web 2.0 in higher education? Procedia - Social and Behavioral Sciences, 1(1), 478-482.

Guitert, M., \& Giménez, F. (2000). Trabajo cooperativo en entornos virtuales de aprendizaje. Aprender en la virtualidad, 10(1), 10-18.

INEC. (2013). Tecnologías de la Información y Comunicaciones (TIC'S) año 2012. Quito: Instituto Nacional de Estadisticas y Censos.

Mora Mora, H., Azorín López, J., Jimeno Morenilla, A., Sánchez Romero, J., Pujol López, F., García Rodríguez, J., . . Saval Calvo, M. (2015). La Web Semántica Como Herramienta para el Apoyo a la Docencia. Departamento de Tecnología Informática y Computación. San Vicente del Raspeig: Universidad de Alicante.

Obando Bastidas, J. (2015). Los OVAS: una experiencia pedagógica que fortalece la formación virtual. Signos universitarios, 2(1), 335-351.

Salinas, J. (2013). ENSENAANZA FLEXIBLE Y APRENDIZAJE ABIERTO, FUNDAMENTOS CLAVE DE LOS PLES. Alcoy: Marfil.

Salinas, M. I. (2011). Entornos virtuales de aprendizaje en la escuela: tipos, modelo didáctico y rol del docente. Buenos Aires: Pontificia Universidad Católica Argentina.

Silva Quiceno, M., \& Sosa, P. (2016). Diseño y Desarrollo de un Objeto Virtual de Aprendizaje para un Curso de Electrónica. INGE CUC, 12(1), 9-20.

Zimmer, M. (2008). Preface: Critical Perspectives on Web 2.0. First Monday, 13(3), 23-27. 